\title{
XXXVI. On the application of Huyghens's principle in physical optics
}

\section{R. Potter Esq. B.A.}

To cite this article: R. Potter Esq. B.A. (1840) XXXVI. On the application of Huyghens's principle in physical optics, Philosophical Magazine Series 3, 17:110, 243-248, DOI: 10.1080/14786444008650168

To link to this article: http://dx.doi.org/10.1080/14786444008650168

册 Published online: 01 Jun 2009.

Submit your article to this journal $[\pi$

Џ Article views: 5

Q View related articles $๘$ 
other arrangements than those which correspond to the maximum of effect, there is no constant relation between different voltaic combinations; we can only compare them, and judge of their relative preferableness by referring them to this maximum of action. We have, by eliminating $z$, the equations

$$
\mathrm{C}(\max )=\frac{\mathrm{A} \sqrt{s}}{2 \sqrt{\lambda \mathrm{L}}}=\frac{\mathrm{A}^{\prime} \sqrt{s^{\prime}}}{2 \sqrt{\lambda^{\prime} \mathrm{L}}}
$$

whence we deduce, by substituting the numerical values above

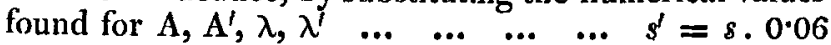
and with reference to the number of pairs $z^{\prime}=z .0 \cdot 6$; that is to say, it requires only a pile of 6 square feet of platina to replace a pile of 100 square feet of copper; or with reference to the number of pairs, 6 pairs of platina, each of a square foot of surface, will produce the same effect as 10 pairs of copper, each of which presents a surface of 10 square feet. This eninent superiority of platina, as in Mr. Grove's combination, is verified by many experiments on a large scale.

XXXVI. On the Application of Huyghens's Principle in Physical Optics. By R. Potтer, Esq., B.A.*

$\mathrm{N}$ the present paper I propose to examine some of the consequences of the method at present followed in developing the results of the undulatory theory of light, which consists in considering elementary waves, having their origin in some previous positions of the main waves, as the cause of these latter in succeeding positions. In the Mémoires de l'Acad. for 1821 and 1822, Fresnel announces this method in the following terms :-

"Application du Principe d'Huygens aux Phénomènes de la Diffraction.

“ Ce principe que me paraît une conséquence rigoureuse de l'hypothèse fondamentale, peut s'énoncer ainsi : Les vibrations d'une onde lumineuse dans chacun de ses points peuvent être regardées comme la somme des mouvemens élémentaires qu'y enverraient au même instant, en agissant isolément, toutes les parties de cette onde considérée dans une quelconque de ses positions antérieures."

Again, in speaking of the analytical process, he says, "La recherche de la loi suivant laquelle leur intensite varierait autour de chaque centre d'ébranlement, présenterait sans doute de grandes difficultés; mais heureusement nous n'avons pas besoin de la connaître; car il est aisé de voir que les effets

* Communicated by the Author. $\mathrm{R} 2$ 
produits par ces rayons se détruisent presque complètement dès qu'ils s'inclinent sensiblement sur la normale, en sorte que ceux qui influent d'une manière appréciable sur la quantité de lumière que reçoit chaque point $P$ peuvent être regardés comme d'égale intensité."

In the application of the above principle, we take the origins of the elementary waves on any proposed surface as that of a reflecting or refracting substance, or an aperture, without its being necessary that this surface should coincide with any one wave surface as it arrives.

There are only a few cases in which the integration for the whole vibration of a particle can be effected directly; in the following, however, the integration involves no difficulty, and they suffice for proving the discordance of the results of the principle with acknowledged facts. They show that the labour which has been expended in investigating more complicated cases, might with ordinary caution have been saved.

The integration is readily performed when a series of plane waves fall on a plane reflector, or an aperture parallel to their surfaces, the reflector or aperture being of one of the circular forms enumerated below, and the particle whose vibration is required, being in the normal to such surfaces through the centre of the circular arcs. These forms comprise a circular aperture of any radius, an annulus contained between two circles, a circular sector, and the quadrilateral figure bounded by two radii, and two circular arcs: this Iatter form approximates to a rectilinear parallelogram, when the angle between the bounding radii is small, and the radii of the circular arcs are large.

The integration for apertures or reflectors of the same forms, is readily performed also when light diverges from a luminous point in the normal line through the centre of the arcs; and the particle whose vibration is required is equally distant from the surface in the same line, and on the opposite or same side with the luminous point, according as an aperture or a reflector is considered.

Let us commence with a series of plane waves falling directly on a quadrilateral aperture KLMN, bounded by the two concentric circular arcs $K N, L M$, and the radii $C M, C L$ containing the angle $\mathrm{M} \mathrm{C} \mathrm{L}=\theta$.

Let $B$ be the position of the particle whose vibration is required situated anywhere in

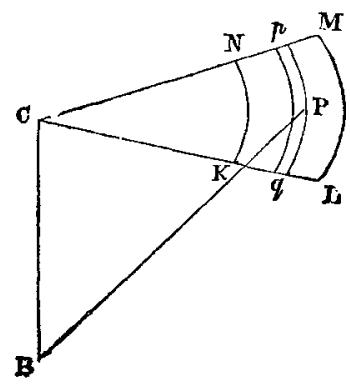


the line $\mathrm{BC}$ which is perpendiculai to the plane of the aperture, and let $\mathrm{C} \mathrm{B}=h$.

Let $p \mathrm{Pq}$ be any element of the aperture, whose breadth $=\delta r$, and distance $\mathbf{C} p$ or $\mathbf{C} q$ from $\mathbf{C}=r$, therefore its distance from $\mathrm{B}=\sqrt{r^{2}+h^{2}}$ and its area $=\theta r \delta r$.

Then, by the principle under discussion, we have the displacement of the particle at $\mathrm{B}$ caused by this element, proportional to

$$
\begin{aligned}
& \frac{\text { area of element }}{\text { distance B P }} \sin \left\{\frac{2 \pi}{\lambda}(v t-\mathrm{B} \mathrm{P})\right\} \\
= & \frac{a \theta r \delta r}{\sqrt{r^{3}+h^{2}}} \sin \left\{\frac{2 \pi}{\lambda}\left(v t-\sqrt{r^{2}+h^{2}}\right)\right\},
\end{aligned}
$$

where $a$ is some number.

Integrating for the whole vibration we have

$$
\begin{aligned}
& a \theta \int_{r} \frac{r}{\sqrt{r^{2}-h^{3}}} \sin \left\{\frac{2 \pi}{\lambda}\left(v t-\sqrt{r^{3}+h^{2}}\right)\right\} \\
& =\frac{a \theta \lambda}{2 \pi} \cos \left\{\frac{2 \pi}{\lambda}\left(r t-\sqrt{r^{2}+h^{2}}\right)\right\}+\mathrm{C} \text { between the limits } \\
& \left.\begin{array}{l}
r=r_{1} \\
r=r_{2}
\end{array}\right\} \\
& =\frac{a \theta \lambda}{\pi} \sin \left\{\frac{\pi}{\lambda}\left(\sqrt{r_{2}^{2}+h^{2}}-\sqrt{\left.r_{1}^{2}+h^{2}\right)}\right\}\right. \text {. } \\
& \sin \left\{\frac{2 \pi}{\lambda}\left(v t-\frac{1}{2} \sqrt{r_{2}^{2}+h^{2}-\frac{1}{2}} \sqrt{r_{1}^{2}+h^{2}}\right)\right\},
\end{aligned}
$$

which gives the intensity of the light at $B$

$$
=\frac{a^{2} \theta^{2} \lambda^{2}}{\pi^{2}} \sin ^{2}\left\{\frac{\pi}{\lambda}\left(\sqrt{r_{2}^{2}+h^{2}}-\sqrt{r_{1}^{2}+h^{2}}\right)\right\} \text {. }
$$

The intensity becomes a maximum and $=\frac{a^{2} \theta^{2} \lambda^{2}}{\pi^{2}}$, or equal to $4 a^{2} \lambda^{2}$ when $\theta=2 \pi$.

If

$$
\sqrt{r_{2}^{3}+h^{2}}-\sqrt{r_{1}^{2}+h^{2}}=\frac{2 n+1}{2} \lambda,
$$

where $n$ is any integer. This equation may be satisfied by an indefinite number of values of $r_{1}$ and $r_{2}$.

When $r_{1}$ and $r_{2}$ are very great, and $h$ small, we have

$$
\begin{aligned}
r_{2}-r_{1}+\frac{h^{8}}{2}\left(\frac{1}{r_{2}}-\frac{1}{r_{1}}\right)+\& \mathrm{c.} . & =\frac{2 n+1}{2} \lambda \\
& =r_{2}-r_{1} \text { nearly. }
\end{aligned}
$$


If now $\theta$ be not large and $r_{2}-r_{1}$ be only a small odd number of multiples of $\frac{\lambda}{2}$, the aperture will be nearly a parallelo. gram, and the maximum intensity $=\frac{a^{2} \theta^{2} \lambda^{2}}{\pi^{2}}$. But if in the original expression we make $r_{1}=0$, the aperture becomes the whole sector, and the maximum intensity, by giving $r_{2}$ the proper value, is $\frac{a^{2} \theta^{2} \lambda^{2}}{\pi^{2}}$. This shows that at however great a distance from $\mathbf{C}$ such a quadrilateral aperture be situated, and however near to $C$ the point $B$ may be, the intensity ought to be the same as for the sector.

The result of the principle is therefore that light ought to bend into the shadows of bodies to an indefinite extent, as sound is known to pass through all apertures, and bend round all obstacles.

It proves that the result Mr. Airy (Tract, page 270.) has obtained by an approximate method is not to be depended upon, and that the objection to the undulatory theory which was believed to have been removed remains in full force.

If it be said that these expressions involving $\lambda^{2}$ as a multiplier, must represent light of very feeble intensity, and therefore insensible, or nearly so, we shall see that we have the same small quantity in the expression for a large circular aperture.

If we make $\theta=2 \pi, r_{2}$ very large, and $r_{1}=0$, we have a large circular aperture, and the intensity

$$
\begin{aligned}
& =4 a^{2} \lambda^{2} \sin ^{2}\left\{\frac{\pi}{\lambda}\left(\sqrt{r_{Q}^{2}+h^{2}}-h\right)\right\} \\
& =4 a^{2} \lambda^{2} \text { when } \sqrt{r_{2}^{2}+h^{2}}-h=\frac{2 n+1}{2} \lambda ;
\end{aligned}
$$

and however great or small $h$ may be, compared with $r_{2}$, we see that there will be a succession of maxima and minima values for different values of $h$. This is at variance with the admitted properties of light, which it is allowed passes through large apertures without any change or diminution, or when diverging from a luminous point follows the law of the inverse square of the distance, except near the boundaries of the shadow.

If we compare the maximum intensity from an annulus, however narrow it may be, and however large the radii, with that from a large circular aperture, we see that they are the same; and the multiplier $\lambda^{2}$ would either show that only a very small quantity of light could pass directly through any large 
circular aperture; or otherwise, if it were maintained that the constant $a$ may be very large; then our former conclusions will be free from any objection that they represent inappreciable quantities of light.

A fact stated by M. Fresnel, which I have confirmed by a severe experimental examination, bears upon the point, of the effect of the limits of apertures. His experiments for the diffraction by a single edge of an opake plate (see the beforementioned memoir, page 429.) were, in fact, made with an aperture generally of a centimeter in breadth, whilst his luminous point and micrometer were in some measures distant about seven metres from each other. Notwithstanding the small breadth of the aperture compared with its distances from the luminous point and micrometer, yet we find him stating that the fringes formed by one edge were not affected by the other edge, and that his measures might be taken as if made with a single edge. The principle under discussion shows, that with the quadrilateral, sectorial or circular forms of apertures, the intensity should depend on the limits, however distant.

The same results arise when we take plane reflectors in place of apertures, only then the point $B$ must be taken on the same side as that on which the waves are incident.

To discuss the second case, let $\mathbf{A}$ be the luminous origin in the line A C B perpendicular to the plane of the aperture, through the centre C. Let $\mathbf{A ~ C}$ $=\mathrm{BC}=h$, and the other parts as in the former figure.

We have now for the displace. ment of the particle at $B$, due to the element $p \mathbf{P} q$,

$$
\begin{aligned}
& \frac{a \theta r \delta r}{\mathrm{AP}+\overline{\mathrm{PB}}} \sin \left\{\frac{2 \pi}{\lambda}(v t\right. \\
& -(\mathrm{AP}+\mathrm{PB}))\}
\end{aligned}
$$

and the whole displacement

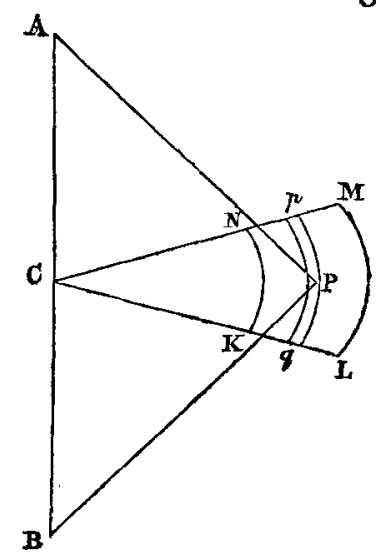

$$
\begin{gathered}
=\frac{a \theta}{2} \int_{r} \frac{r}{\sqrt{r^{2}+h^{2}}} \sin \left\{\frac{2 \pi}{\lambda}\left(v t-2 \sqrt{\left.r^{2}+h^{2}\right)}\right\}\right. \\
=\frac{a \theta \lambda}{8 \pi} \cos \left\{\frac{2 \pi}{\lambda}\left(v t-2 \sqrt{r^{2}+h^{2}}\right)\right\}+C \text { between the } \\
\text { limits } \left.\begin{array}{rl}
r & =r_{1} \\
r & =r_{2}
\end{array}\right\}
\end{gathered}
$$


248 On Huyghens's Principle applied to Physical Optics.

$$
\begin{aligned}
&=\frac{a \theta \lambda}{4 \pi} \sin \left\{\frac{2 \pi}{\lambda} \sqrt{r_{2}^{2}+h^{2}}-\left(\sqrt{\left.r_{1}^{2}+h^{2}\right)}\right\}\right. \\
& \sin \left\{\frac{2 \pi}{\lambda}\left(v t-\sqrt{r_{2}^{9}+h^{2}}-\sqrt{\left.r_{1}^{2}+h^{2}\right)}\right\}\right.
\end{aligned}
$$

and the intensity

$$
=\frac{a^{2} \theta^{2} \lambda^{2}}{4^{2} \pi^{2}} \sin ^{2}\left\{\frac{2 \pi}{\lambda}\left(\sqrt{r_{2}^{2}+h^{2}}-\sqrt{r_{1}^{2}+h^{2}}\right)\right\} \text {. }
$$

As in the former case, when we take $h$ small and $r_{1}, r_{2}$ very large, with $\theta$ small, so as to form a quadrilateral aperture approximating very nearly to a parallelogram, we arrive at the same conclusion, that light, according to the principle under discussion, ought to pass through apertures, however obliguely situated with respect to its direction, and diverge into the shadow to an indefinite extent; the maximum intensity at $B$, being the same as if the aperture were the whole sector, and this holding, however near $A$ and $B$ may be to $C$.

If we take $A=2 \pi$ and take $r_{2}$ very large, whilst $r_{1}$ is small, we have the case of the intensity in the centre of the shadow of a small circular disc, which it was found by approximate methods, ought to be the same as if the light passed uninterrupted; and M. Arago, having tried the experiment, found the result to accord. The complete investigation gives the intensity

$$
=\frac{\alpha^{2} \lambda^{2}}{2^{2}} \sin ^{2}\left\{\frac{2 \pi}{\lambda} \sqrt{\left(r_{2}^{2}+h^{2}\right.}-\sqrt{\left.r_{1}^{2}+h^{2}\right)}\right\},
$$

which goes through a series of maxima and minima values for different values of $h$, when $r_{1}$ and $r_{2}$ are given; and not an uniform or slowly diminishing intensity along this line, as found by the approximate discussion.

There is another case which places the absurdity of the principle in a very striking point of view, which is the case of a large circular aperture, then $r_{1}=0$, and we have the intensity

$$
=\frac{a^{2} \lambda^{2}}{2^{2}} \sin ^{2}\left\{\frac{2 \pi}{\lambda}\left(\sqrt{r_{2}^{2}+h^{2}}-\lambda\right)\right\}:
$$

the maximum intensity is here dependent on $h$ for its position, but not for its magnitude; that is, the maximum intensity at $B$ is $\frac{a^{2} \lambda^{2}}{2^{2}}$, however near A and $B$ may be together, or however distant ; contrary to the received and demonstrated principle, that the intensity of light diverging from a luminous origin varies as the inverse square of the distance.

Queen's College, Sept. 1840. 